\title{
Microwave-induced thermal escape in Josephson junctions
}

\author{
N. Grønbech-Jensen ${ }^{1}$, M. G. Castellano ${ }^{2}$, F. Chiarello ${ }^{2}$, G. Torrioli ${ }^{2}$, M. Cirillo ${ }^{3}$, L. \\ Filippenko ${ }^{3,4}$, R. Russo ${ }^{3}$, and C. Cosmelli ${ }^{5}$ \\ ${ }^{1}$ Department of Applied Science, University of California, Davis, \\ California 95616 \\ ${ }^{2}$ IFN-CNR, via Cineto Romano 42, I-00156 Rome, Italy \\ ${ }^{3}$ Department of Physics and INFM, University of Rome "Tor Vergata", \\ I-00133 Rome, Italy \\ ${ }^{4}$ Institute of Radio Engineering and Electronics, Mokhovaya, \\ 103907 Moscow, Russia \\ ${ }^{5}$ Department of Physics and INFN, University of Rome, "La Sapienza", \\ I-00185 Rome, Italy
}

(November 23, 2018)

\begin{abstract}
We investigate, by experiments and numerical simulations, thermal activation processes of Josephson tunnel junctions in the presence of microwave radiation. When the applied signal resonates with the Josephson plasma frequency oscillations, the switching current may become multi-valued in a temperature range far exceeding the classical to quantum crossover temperature. Plots of the switching currents traced as a function of the applied signal frequency show very good agreement with the functional forms expected from Josephson plasma frequency dependencies on the bias current. Throughout, numerical simulations of the corresponding thermally driven classical Josephson junction model show very good agreement with the experimental data.
\end{abstract}


The Josephson tunnel junction is an intriguing solid state physics system due to the macroscopic quantum nature of the variables describing the governing equations [1]; irradiation of junctions with microwave (ac) radiation has produced a number of significant nonlinear phenomena such as chaos and phase-locking observed both in experiments and theoretical models. These phenomena have been investigated for almost four decades [2,3] and successful applications have significantly benefited from complete theoretical understanding of the relevant dynamics.

Measurements of the escape statistics from the zero-voltage state in Josephson junctions have been conducted extensively over the years, successfully confirming consistency with the classic Kramers model for thermally activated escape from a potential well $[4,5]$. Escape measurements represent a powerful tool for probing the nature of potentials and they have also been employed to discriminate between classical and quantum behavior of the junctions. Work performed in the past two decades [6-9] has contributed to generating general interest toward Josephson junction systems and represented the background for recent work in the field of quantum coherence and quantum computing [11-13]. Several groups have experimentally investigated the effects of applying ac signals to the junction during measurements of escape out of the Josephson washboard potential well $[6,7,10]$. Applying an ac field to a low-temperature system has been reported to produce anomalous switching distributions with signatures of two, or more, distinct dc bias currents for which switching is likely. These measurements have been interpreted as a signature of the ac field aiding the population of multiple quantum levels in a junction, thereby leading to enhancement of the switching probability for bias currents for which the corresponding quantum levels match the energy of the microwave photons.

In this letter we report on a systematic investigation of the escape properties of a Josephson tunnel junction subject to external microwave radiation and interpret the results classically. We have carried out an extensive experimental investigation varying the temperature of the samples from $0.36 K$ up to $1.6 K$. The results are compared with numerical simulations of the well-known classical RSCJ model [2] for a Josephson junction with appropriate per- 
turbations terms reflecting the presence of damping, thermal noise, dc and ac bias currents.

Figure 1 illustrates the process under investigation: in the classical one-degree-offreedom single-particle washboard potential of the Josephson junction [6], thermal excitations (shaded in the sketch) of energy $k_{B} T$ and the energy $E_{a c}$ of forced oscillations due to microwave radiation, can cause the particle to escape from the potential well. This process can be traced by sweeping the current-voltage characteristics of the Josephson junction periodically. Escape from the potential well corresponds to an abrupt transition from the top of the Josephson-current zero-voltage state to a non-zero voltage state. The statistics of the switching events, in the absence of time-varying perturbations, have been shown to be consistent with Kramers' model [4] for thermal escape from a one-dimensional potential. Since the thermal equilibrium Kramers model does not include the effect of non-equilibrium force terms, the results of the switching events generated by the presence of a microwave radiation on a Josephson junction can be investigated, in a thermal regime, only by a direct numerical simulation of the governing equation,

$$
\frac{\hbar C}{2 e} \frac{d^{2} \varphi}{d t^{2}}+\frac{\hbar}{2 e R} \frac{d \varphi}{d t}+I_{c} \sin \varphi=I_{d c}+I_{a c} \sin \omega_{d} t+N(t)
$$

Here, $\varphi$ is the phase difference of the quantum mechanical wave functions of the superconductors defining the Josephson junction, $C$ is the magnitude of junction capacitance, $R$ is the model shunting resistance, and $I_{c}$ is the critical current, while $I_{d c}$ and $I_{a c} \sin \omega_{d} t$ represent, respectively, the continuous and alternating bias current flowing through the junction. The microwave energy sketched in Figure 1 will then be $\propto I_{a c}^{2}$. The term $N(t)$ represents the thermal noise-current due to the resistor $R$ given by the thermodynamic dissipation-fluctuation relationship [14]

$$
\begin{aligned}
\langle N(t)\rangle & =0 \\
\left\langle N(t) N\left(t^{\prime}\right)\right\rangle & =2 \frac{k_{B} T}{R} \delta\left(t-t^{\prime}\right),
\end{aligned}
$$

with $T$ being the temperature. The symbol, $\delta\left(t-t^{\prime}\right)$, is the Dirac delta function. Current and time are usually normalized respectively to the Josephson critical current $I_{c}$ and to 
$\left(\omega_{0}\right)^{-1}$, where $\omega_{0}=\sqrt{2 e I_{c} / \hbar C}$ is the Josephson plasma frequency. With this normalization, the coefficient of the first-order phase derivative becomes the normalized dissipation $\alpha=$ $\hbar \omega_{0} / 2 e R I_{c}$. It is also convenient to scale the energies to the Josephson energy $E_{J}=I_{c} \hbar / 2 e=$ $I_{c} \Phi_{0} / 2 \pi$, where $\Phi_{0}=h / 2 e=2.07 \cdot 10^{-15} \mathrm{~Wb}$ is the flux-quantum. Thus, the set of equations (1-3) can be expressed in normalized form as

$$
\begin{aligned}
\ddot{\varphi}+\alpha \dot{\varphi}+\sin \varphi & =\eta+\eta_{d} \sin \Omega_{d} \tau+n(\tau) \\
\langle n(\tau)\rangle & =0 \\
\left\langle n(\tau) n\left(\tau^{\prime}\right)\right\rangle & =2 \alpha \theta \delta\left(\tau-\tau^{\prime}\right),
\end{aligned}
$$

where $\theta=\frac{k_{B} T}{E_{J}}$ is the normalized temperature.

Figure 2 shows the results of numerical simulations of escape in a system with $\alpha=$ $0.00845, \theta=4.76 \cdot 10^{-4}$, and continuous bias sweep rate $\frac{d \eta}{d \tau}=2.1 \cdot 10^{-8}$. These parameters have been chosen in correspondence with the experimental measurements discussed below. The resulting switching distributions (each corresponding to 10000 events), obtained for different values of the normalized drive frequency, are shown as a function of the continuous bias, and we clearly observe that secondary peaks in the distribution may appear, along with the primary peak corresponding to the unperturbed $\left(\eta_{d}=0\right)$ switching distribution. The bias location of the secondary peak of each distribution is pointing by a dotted line to the intersection (marked with $\bullet$ ) with the driving frequency on the top plot of Figure 2. Also on the top plot, we have shown the linear plasma resonance curve, $\Omega_{p}=\left(1-\eta^{2}\right)^{1 / 4}$, as a solid line, and we observe that the numerically obtained switching distribution peaks are closely aligned with the linear plasma resonance near the driving frequency.

Notice that the values of the ac drive amplitude $\eta_{d}$ were tuned (as indicated at the right margin of each plot) in order to achieve the resonant energy necessary to escape the potential well. Naturally, the lowest values of dc bias current need larger ac amplitudes to produce a significant number of switching events. The fact that the data points on the top graph are consistently below the predicted (linear) resonance curve can be directly attributed to the anharmonicity of the Josephson potential for large bias currents [15]. 
Thermal escape properties of the Josephson junction, as described by Eqs. (4-6), depend strongly on the value of the normalized frequency $\Omega_{d}$. We have repeated the same numerical procedure as described in Figure 2 for drive frequencies equal to sub- and superharmonics of the frequencies leading to the direct resonances observed above. Specifically, Figure 3 reports results for driving frequencies $\Omega_{d}=q \Omega_{p}$ with $q=\frac{1}{5}, \frac{1}{4}, \frac{1}{3}, \frac{1}{2}, 1,2$ (notice that $q=1$ is the case shown in Figure 2). The numerical results for both super- and subharmonics are shown in Figure 3 (circles o). In this figure we see that, even when driving at subharmonics or superharmonics, the positions of the secondary switching peaks follow closely what can be expected from the plasma resonance dependence on the bias current. It is worth noting that the escape histograms often revealed multi-peaked distributions with more than two peaks. An example of this is shown in the lowest plot of Figure 2 where we see two peaks close to each other and another well separated on the left. These are merely an expression of the fact that the variation in the bias current is tuning the plasma frequency, which in turn may find some rational resonance with the applied frequency, thereby enhancing the induced amplitude of oscillation in the junction and increase the probability for escape.

Complementary experiments were performed on Josephson tunnel junctions fabricated according to classical Nb-NbAlOx-Nb procedures [16]. The samples had very good currentvoltage characteristics and magnetic field diffraction patterns. The junctions were cooled in a ${ }^{3}$ He refrigerator (Oxford Instruments Heliox system), providing temperatures down to $360 \mathrm{mK}$. The microwave radiation, brought to the chip-holders by a coax cable, was coupled capacitively to the junctions. The junction had a maximum critical current of $143 \mu A$ and a total capacitance of $6 p F$ from which we estimate a plasma frequency of $42.5 \mathrm{GHz}$. From this value of the (unbiased) plasma frequency the classical to quantum crossover temperature [17] $T^{*}=\left(\hbar \omega_{0} / 2 \pi k_{B}\right)=320 m K$ between classical thermal and quantum mechanical behavior can be estimated. The sweep rate of the continuous current $I_{d c}$ was $\dot{I}_{d c}=800 \mathrm{~mA} / \mathrm{s}$, and we verified that the experiment was being conducted in adiabatic conditions [8]. At the temperature $T=1.6 \mathrm{~K}$ the junction had a Josephson energy $E_{J}=46.4 \cdot 10^{-21} \mathrm{~J}$, and effective resistance $R=74 \Omega$. We have gathered data in the temperature range of $360 \mathrm{mK}-1.6 \mathrm{~K}$, but 
we will here show the results for $T=1.6 K$. As mentioned above, simulations were performed on the basis of these real system parameters. The evaluation of the dissipation parameter was based on the hysteresis of the current-voltage characteristics of the junctions $[2,3]$; we anticipate that the dissipation parameter used in the simulations may not completely represent the experimental dissipation.

Figure 4 displays the experimentally obtained complementary results to Figure 2 . It is clear that also the experiments exhibit close agreement between the bias location of the secondary switching current and the plasma frequency resonance when the value of drive frequency is near the plasma frequency resonance. We note that the level of the applied power is not a simple linear function (like in Figure 2) because resonances in the coupling system caused a slightly irregular coupling from the microwaves to the junction. In Figure 4 each plot is obtained by recording 2000 switching events.

Similarly to the simulations, we have also conducted escape measurements using subharmonic microwave radiation. These data are shown in Figure 3 as squares ( $\square$ ) alongside the corresponding simulation results discussed above. In the experiments, as in the simulations, we see that the position of the secondary peak follows closely what would be expected from the plasma frequency dependence on the dc current bias. We emphasize that no fitting was used in Figure 3 for neither numerical/experimental data nor the plasma resonance curves. Data obtained at different temperatures in the range $(360 m K-1.6 K)$ showed features identical to those of Figures 2-4 for both simulations and experiments. As was the case in the simulations, we also frequently observed switching distributions with three peaks in the experiments.

In conclusion, we have reported on numerical simulations and experiments on ac-driven, thermal escape of a classical particle from a one-dimensional potential (the Josephson washboard potential) well. We have found that resonant coupling (harmonic or subharmonic) between the applied microwaves and the plasma resonance frequency of the junction provides an enhanced opportunity for escape, and we have directly observed the signatures of such microwave-induced escape distributions in the form of anomalous multi-peaked escape 
statistics. The straightforward agreement between the classical hypothesis of anomalous distributions being directly produced by ac-induced resonances, the results of numerical simulations of the classical pendulum model of a Josephson junction, and actual Josephson junction experiments indicate a consistent interpretation of ac-induced anomalous multipeaked switching distributions in the classical regime of Josephson junctions.

It is noted that previous experimental work on ac-induced escape distributions obtained at temperatures below $T^{*}$ is consistent with the observations presented here. Those experiments have produced ac-induced peaks in the observed switching distributions, and the relevant peaks are located alongside the expected classical plasma resonance curve, as we have also found here. An important observation is that the microwave-radiation frequency necessary for populating an excited quantum level $\left(\hbar \omega_{d}\right)$ in a quantum oscillator coincides with the classical resonance frequency of the corresponding classical oscillator. Thus, the switching distributions obtained from classical and quantum mechanical oscillators may exhibit the same microwave induced multi-peak signatures, which in the classical interpretation is merely due to resonant nonlinear effects. The experimental and numerical evidence herein reported, along with data existing below $T^{*}[10]$, point again to the Josephson junction as an ideal vehicle for probing relevant issues in fundamental physics [18].

This work was supported in part by the Computational Nanoscience Group, Motorola, Inc. We are happy to acknowledge Prof. Alan Laub for a careful reading of the manuscript. 


\section{REFERENCES}

[1] P. W. Anderson, in Lectures on the Manybody Problem, Vol. 2 (E. R. Caianiello ed., Academic Press, NY,1964), p.132.

[2] A. Barone and G. Paternó, Physics and Applications of the Josephson Effect (Wiley, New York, 1982).

[3] T. Van Duzer and C. W. Turner, Principles of Superconductive Devices and Circuits , 2nd ed.(Prentice-Hall, New York, 1998)

[4] H. A. Kramers, Physica 7, 284 (1940).

[5] T. A. Fulton and L. N. Dunkelberger, Phys. Rev. B 9, 4760 (1974).

[6] J. M. Martinis, M. H. Devoret, and J. Clarke, Phys. Rev. Lett. 55, 1543 (1985).

[7] M. H. Devoret, J. M. Martinis, and J. Clarke, Phys. Rev. Lett. 55, 1908 (1985).

[8] P. Silvestrini, V. G. Palmieri, B. Ruggiero, and M. Russo, Phys. Rev. Lett. 79, 3046 (1997).

[9] B. Ruggiero, M. G. Castellano, G. Torrioli, C. Cosmelli, F. Chiarello, V. G. Palmieri, C. Granata, and P. Silvestrini, Physical Review B59, 177 (1999).

[10] A. Wallraff, T. Duty, A. Lukashenko, and A. V. Ustinov, Phys. Rev. Lett. 90, 037003 (2003).

[11] J. M. Martinis, S. Nam, and J. Aumentado, Phys. Rev. Lett. 89, 117901 (2002).

[12] Y. Lu, S. Han, Xi Chu, S. Chu, Z. Wang, Science 296, 889 (2002).

[13] A. J. Berkley, H. Xu, M. A. Gubrud, R. C. Ramos, J. R. Anderson, C. Lobb, and F. C. Wellstood, Physical Review B68, 060502 (2003).

[14] See, e.g., G. Parisi, Statistical Field Theory (Addison-Wesley, 1988).

[15] N. Grønbech-Jensen et al., to be published. 
[16] S. Morohashi and S. Hasuo, J. Appl. Phys. 61, 4835 (1987).

[17] I. Affleck, Phys. Rev. Lett. 46, 388 (1981).

[18] V. Averbukh and N. Moiseyev, Physical Review A57, 1345 (1998). 


\section{FIGURES}

FIG. 1. Sketch of the physical phenomenon under investigation: a driven oscillation energy $E_{a c}$ superimposed onto thermal excitations, may cause a particle to escape a washboard potential.

FIG. 2. Simulated switching distributions, $\rho(\eta)$, for the ac-driven junction model obtained for increasing values of the drive frequency. The frequency data points in the uppermost plot are relative to the position of the secondary peak in the plots. Normalized temperature is $\theta=4.76 \cdot 10^{-4}$, bias sweep rate is $\dot{\eta}=2.1 \cdot 10^{-8}$, and damping coefficient is $\alpha=0.00845$. Solid curve in uppermost graph represents the linear plasma resonance $\Omega_{p}$.

FIG. 3. The functional dependencies of the driving frequency upon the location of the secondary peak in $\rho(\eta)$ obtained for subharmonic and harmonic pumping. Circles (o) represent numerical results and squares $(\square)$ experimental data. Parameters are as given in Figure 2. Solid curves represent $q \Omega_{p}$, for different $q$, as indicated.

FIG. 4. The experimental complement to Figure 2 for a thermodynamic temperature $(1.6 K)$ equivalent to the numerical normalized value. 


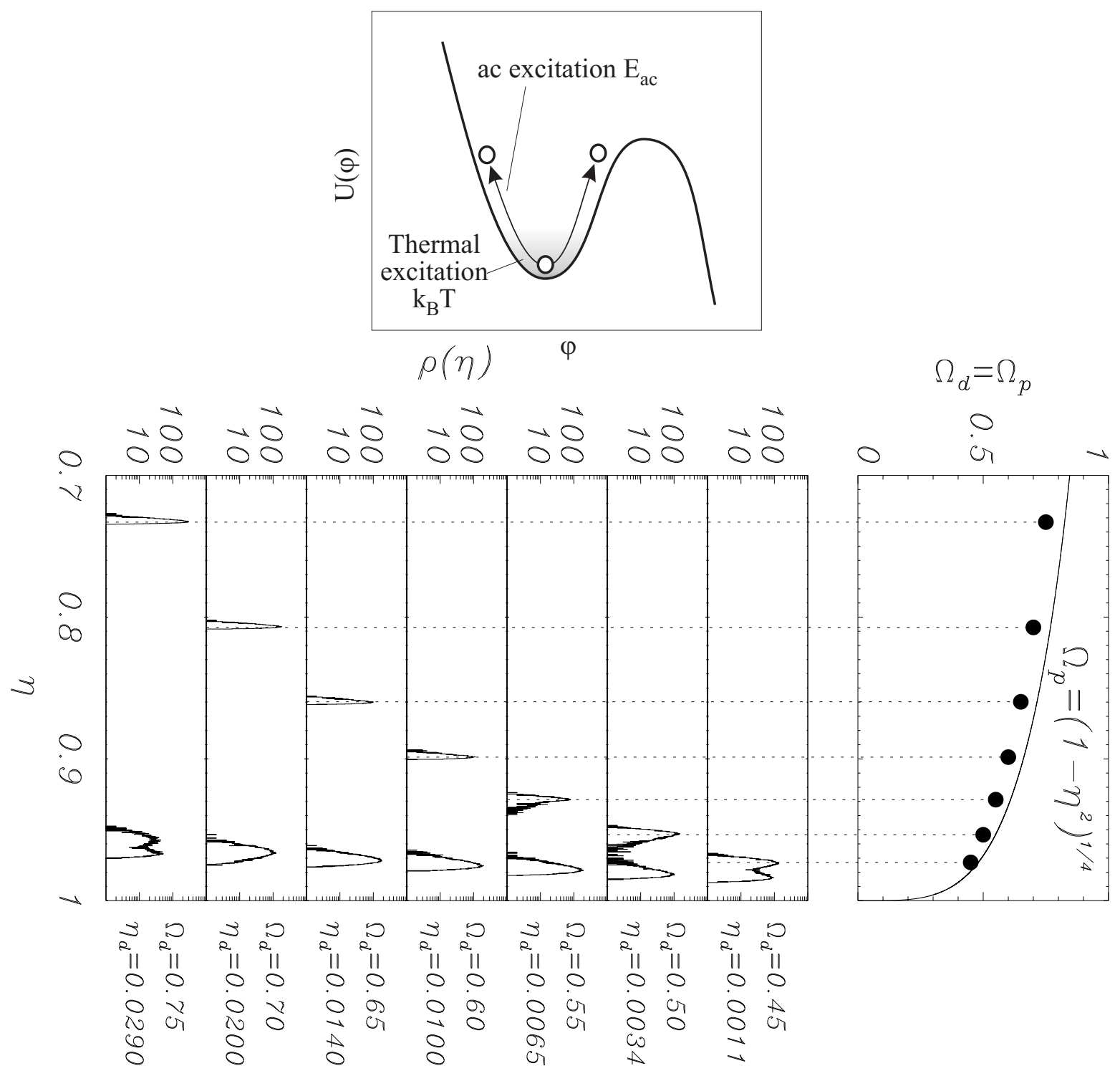




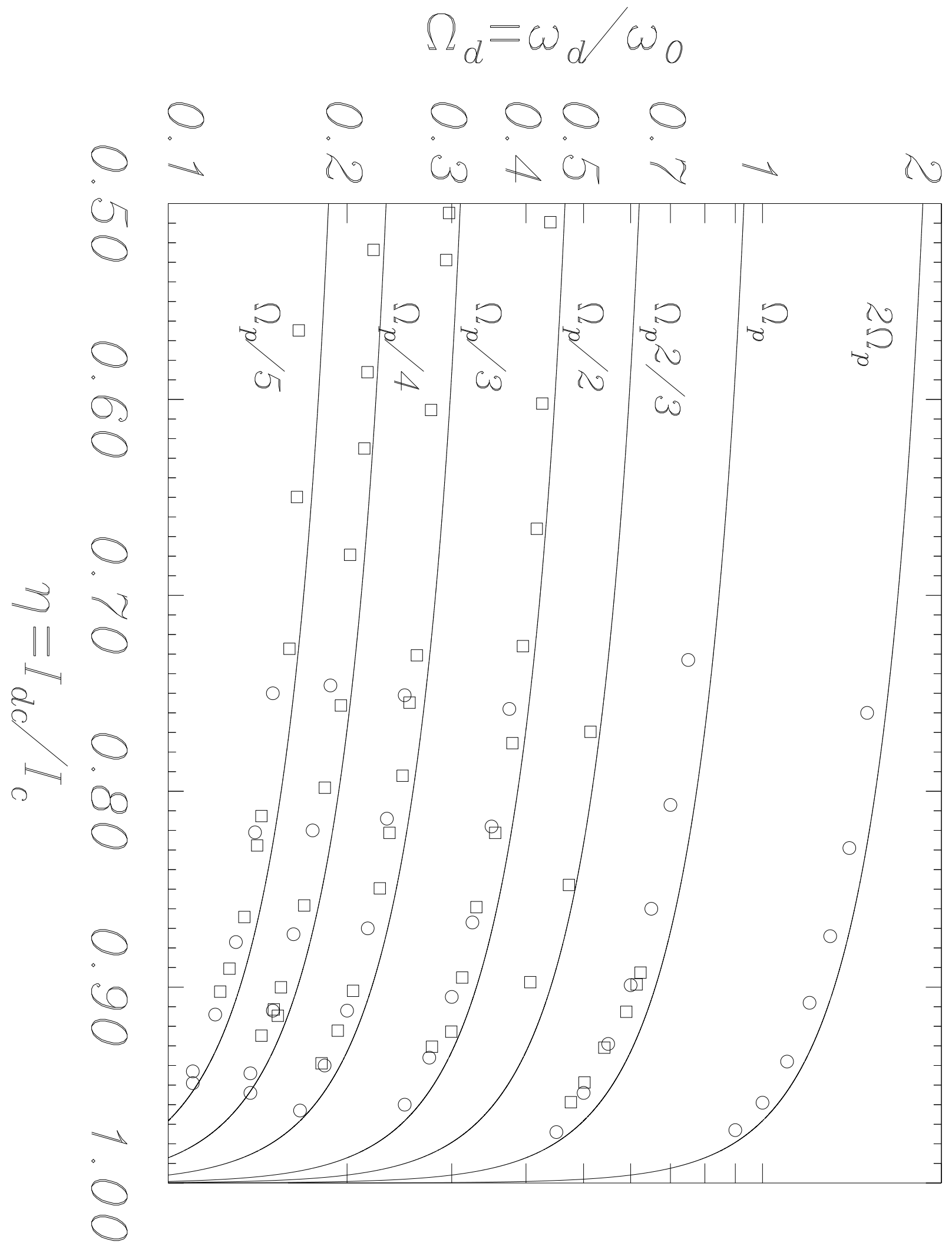




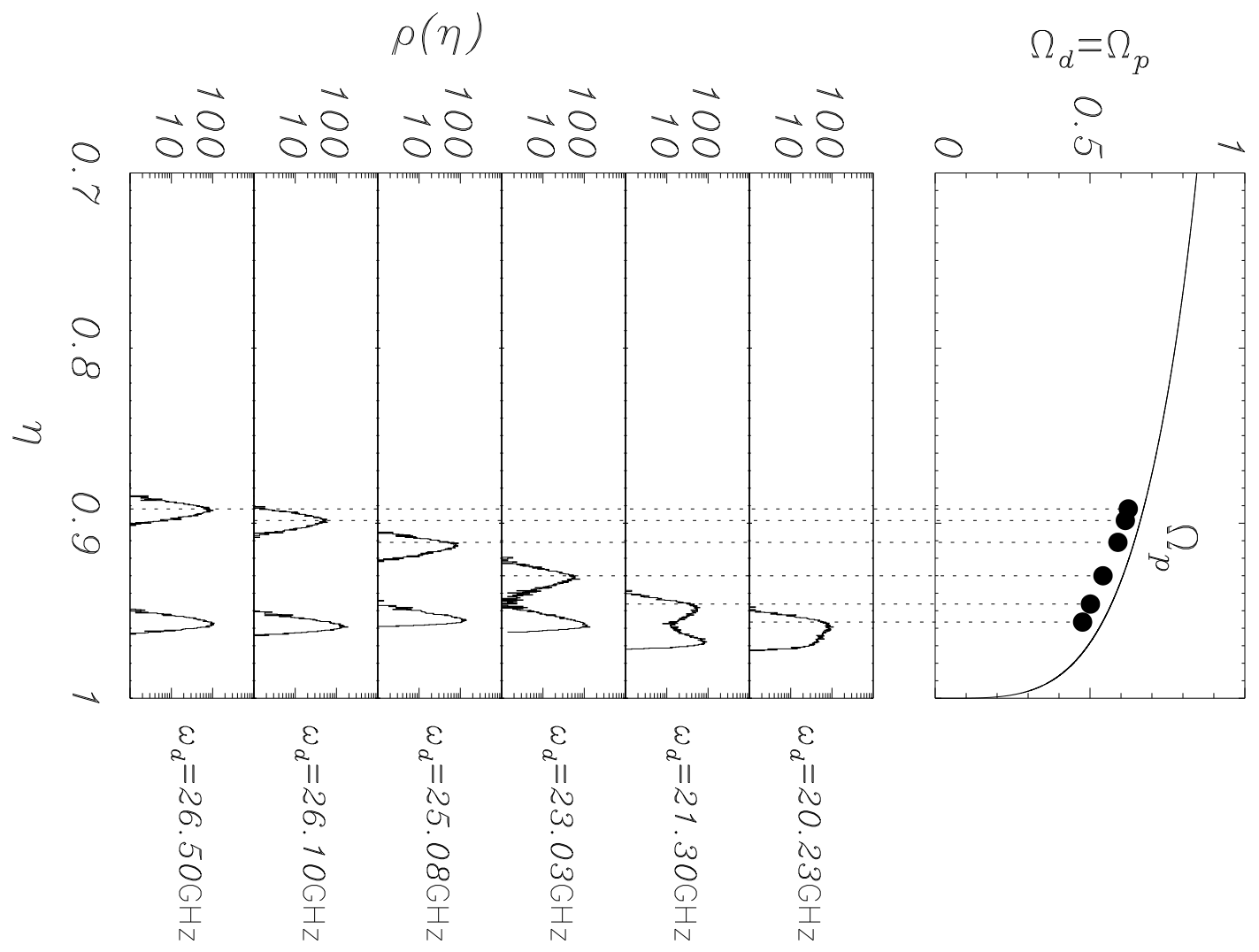

